BULLETIN OF THE POLISH

ACADEMY OF SCIENCES

MATHEMATICS

Vol. 65, No. 1, 2017

\title{
Metrization in small and large scale structures
}

by

\author{
Takahisa MIYATA
}

Presented by Czesław BESSAGA

\begin{abstract}
Summary. Given a topological structure and a coarse structure on a set, N. Wright gave a necessary and sufficient condition for the set to have a metric inducing simultaneously both the structures. We use the idea of the Alexandroff and Urysohn metrization theorem for topological spaces, to investigate a simultaneous metrization problem for a set with a uniform (and topological) structure and a coarse structure. In particular, we prove that given two metrics $d_{U}$ and $d_{C}$ on a set $X$ such that the uniform (topological) structure induced by $d_{U}$ is compatible in some sense with the coarse structure induced by $d_{C}$, there exists a metric $d$ on $X$ which is isometric to $d_{U}$ in a small scale and to $d_{C}$ in a large scale. We then apply this idea to show that if, in addition, the uniform space has uniform dimension 0 and the coarse space has asymptotic dimension 0 , then there exists an ultrametric $d$ on $X$ which is isometric to $d_{U}$ in small scale and to $d_{C}$ in large scale.
\end{abstract}

1. Introduction. A metric on a set $X$ induces a topological structure, a uniform structure and a coarse structure. Given one of the structures on $X$, a metrization problem is to find a metric inducing the structure. N. Wright [11] considered a simultaneous metrization problem for a set $X$ with both a topological structure and a coarse structure, which means finding a metric on $X$ inducing the topological structure and the coarse structure. Wright proved that for every set $X$ equipped with metrics $d_{T}$ and $d_{C}$ such that the coarse structure induced by $d_{C}$ is compatible with the topology induced by $d_{T}$, i.e., there is an open entourage containing the diagonal, there exists a metric $d$ on $X$ which induces the topology induced by $d_{T}$ and is quasi-isometric to $d_{C}$.

On the other hand, the metrization theorem of Alexandroff and Urysohn [1], which states that every normal sequence of covers of a topological

2010 Mathematics Subject Classification: Primary 54E35; Secondary 54F45.

Key words and phrases: metrization, large scale structure, small scale structure, normal sequence.

Received 20 August 2016; revised 12 March 2017.

Published online 15 May 2017. 
space $X$ (satisfying some reasonable condition) admits a metric on $X$ inducing the topology, has proven to be useful in studying metric properties. In this paper, using the idea of Alexandroff and Urysohn, we introduce a new approach to the simultaneous metrization problem for a set with a uniform structure (or a topological structure) and a coarse structure.

We define the notion of normal sequence, which is applicable to both small scale and large scale. More precisely, a normal sequence on a set $X$ is a family $\left\{\mathcal{U}_{i}: i \in \mathbb{Z}\right\}$ of covers of $X$ such that $\mathcal{U}_{i+1}$ star-refines $\mathcal{U}_{i}$ and some other conditions are satisfied. Simultaneous metrization of a set with a uniform structure (or a topological structure) and a coarse structure is considered in the following two theorems.

TheOREM 1.1. Let $X$ be a set with a uniform structure and a coarse structure. Then $X$ is simultaneously metrizable if and only if there exists a normal sequence $\mathfrak{U}=\left\{\mathcal{U}_{i}: i \in \mathbb{Z}\right\}$ on $X$ such that

(1) the family $\left\{\mathcal{U}_{i}: i \leq 0\right\}$ generates the coarse structure, and

(2) the family $\left\{\mathcal{U}_{i}: i \geq 0\right\}$ generates the uniform structure.

THEOREM 1.2. Let $X$ be a set with a topological structure and a coarse structure. Then $X$ is simultaneously metrizable if and only if there exists a normal sequence $\mathfrak{U}=\left\{\mathcal{U}_{i}: i \in \mathbb{Z}\right\}$ on $X$ such that

(1) the family $\left\{\mathcal{U}_{i}: i \leq 0\right\}$ generates the coarse structure, and

(2) for each $x \in X$, the family $\left\{\operatorname{St}\left(x, \mathcal{U}_{i}\right): i \geq 0\right\}$ is a base at $x$ for the topological structure.

We then consider a situation where a set is given two metrics, one of which gives a uniform structure and the other a coarse structure. Taking the Alexandroff and Urysohn approach by normal sequences, we prove that if the two structures are compatible in some natural sense, then there exists a metric which is isometric to the given metrics in the corresponding scales. Here we need the notion of small scale Lipschitz equivalence between metrics, which is dual to the notion of quasi-isometric map.

TheOREM 1.3. Let $X$ be a set with metrics $d_{S}$ and $d_{L}$. If the uniform structure $\mu$ induced by $d_{S}$ is compatible with the coarse structure $\lambda$ induced by $d_{L}$, then there exists a metric $d$ such that the metric space $(X, d)$ is small scale Lipschitz equivalent to $\left(X, d_{S}\right)$ and quasi-isometric to $\left(X, d_{L}\right)$.

An analogous problem is considered for a set with two metrics, one of which gives a topological structure and the other a coarse structure. Here we use the notion of lipeomorphism, which means a local Lipschitz equivalence. The following theorem improves the result of Wright by showing that the resulting metric is lipeomorphic to the given metric. 
TheOREM 1.4. Let $X$ be a set with metrics $d_{T}$ and $d_{L}$. If the topological structure $\tau$ induced by $d_{T}$ is compatible with the large scale structure $\lambda$ induced by $d_{L}$, then there exists a metric $d$ such that $(X, d)$ is lipeomorphic to $\left(X, d_{T}\right)$ and quasi-isometric to $\left(X, d_{L}\right)$.

A separable metric space $(X, d)$ has covering dimension 0 if and only if there exists an ultrametric $\rho$ on $X$ such that the identity map $1_{X}:(X, \rho) \rightarrow$ $(X, d)$ is a homeomorphism (see [5], [9]); and a metric space $(X, d)$ has uniform dimension 0 (resp., asymptotic dimension 0 ) if and only if there exists an ultrametric $\rho$ on $X$ such that the identity map $1_{X}:(X, \rho) \rightarrow(X, d)$ is a uniform equivalence (resp., coarse equivalence) (see [3], [7]). As an application of the above results, we consider simultaneous ultrametrization for a set with a 0-dimensional uniform structure and a 0-dimensional coarse structure. We prove the following two theorems.

THEOREM 1.5. Let $X$ be a set with metrics $d_{S}$ and $d_{L}$, and equip it with the uniform structure $\mu$ induced by $d_{S}$ and the coarse structure $\lambda$ induced by $d_{L}$. Suppose that $\mu$ is compatible with $\lambda$. Then the uniform space $(X, \mu)$ has uniform dimension 0 and the coarse space $(X, \lambda)$ has asymptotic dimension 0 if and only if $X$ admits an ultrametric $d$ such that $(X, d)$ is small scale Lipschitz equivalent to $\left(X, d_{S}\right)$ and quasi-isometric to $\left(X, d_{L}\right)$.

TheOREM 1.6. Let $X$ be a set with metrics $d_{T}$ and $d_{L}$, and equip it with the topological structure $\tau$ induced by $d_{T}$ and the large scale structure $\lambda$ induced by $d_{L}$. Then the topological space $(X, \tau)$ has covering dimension 0 and the coarse space $(X, \lambda)$ has asymptotic dimension 0 if and only if $X$ admits an ultrametric $d$ such that $(X, d)$ is lipeomorphic to $\left(X, d_{T}\right)$ and quasi-isometric to $\left(X, d_{L}\right)$.

Throughout the paper, for uniform structures, we use the definition of uniformity by J. R. Isbell [6], and for coarse strucures, we use the definition by J. Dydak and C. S. Hoffland [4], who introduced the notion of large scale structure using uniformly bounded families and showed its equivalence to the notion of coarse structure. We use the terms "small scale structure" and "large scale structure" for uniform structure and coarse structure, respectively.

2. Preliminaries. Let $X$ be a set, and let $\mathcal{U}, \mathcal{V}$ be families of subsets of $X$. For each $A \subset X$, let $\operatorname{St}(A, \mathcal{U})=\bigcup\{U \in \mathcal{U}: U \cap A \neq \emptyset\}$, St $\mathcal{U}=$ $\{\operatorname{St}(U, \mathcal{U}): U \in \mathcal{U}\}$, and $\operatorname{St}(\mathcal{U}, \mathcal{V})=\{\operatorname{St}(U, \mathcal{V}): U \in \mathcal{U}\}$. The family $\mathcal{U}$ is called a refinement of $\mathcal{V}$, in notation $\mathcal{U}<\mathcal{V}$, if for each $U \in \mathcal{U}$ there is $V \in \mathcal{V}$ such that $U \subset V$, and $\mathcal{U}$ is a star refinement of $\mathcal{V}$, written $\mathcal{U}<^{*} \mathcal{V}$, if St $\mathcal{U}<\mathcal{V}$. Let $\mathcal{U} \wedge \mathcal{V}=\{U \cap V: U \in \mathcal{U}, V \in \mathcal{V}\}$. If $A=\{x\}$, we write $\operatorname{St}(x, \mathcal{U})$ for $\operatorname{St}(\{x\}, \mathcal{U})$. Two points $x$ and $y$ in $X$ are said to be $\mathcal{U}$-near, in symbols $(x, y)<\mathcal{U}$, if $x, y \in U$ for some $U \in \mathcal{U}$. Write $(x, y) \nless \mathcal{U}$ if $x$ and $y$ are not $\mathcal{U}$-near. 
Let $d$ be a metric on a set $X$. For each $r>0$, let $\mathrm{B}_{d}(x, r)=\{y \in X$ : $d(x, y)<r\}$ and $\overline{\mathrm{B}}_{d}(x, r)=\{y \in X: d(x, y) \leq r\}$. For every subset $A$ of $X$, let $\operatorname{diam}_{d}(A)$ denote the diameter of $A$. The mesh of $\mathcal{U}$, denoted $\operatorname{mesh}_{d}(\mathcal{U})$, is defined as $\sup \left\{\operatorname{diam}_{d}(U): U \in \mathcal{U}\right\}$, and the Lebesgue number of $\mathcal{U}$, denoted $\operatorname{Leb}_{d}(\mathcal{U})$, is the supremum of the positive real numbers $r$ such that for every $A \subseteq X$ with $\operatorname{diam}_{d} A \leq r$ there exists $U \in \mathcal{U}$ with $A \subset U$. A cover $\mathcal{U}$ of $X$ is said to be uniform if $\operatorname{Leb}_{d}(\mathcal{U})>0$, and uniformly bounded if $\operatorname{mesh}_{d}(\mathcal{U})<\infty$.

A function $f:\left(X, d_{X}\right) \rightarrow\left(Y, d_{Y}\right)$ between metric spaces is Lipschitz if there exists $c>0$ such that $d_{Y}\left(f(x), f\left(x^{\prime}\right)\right) \leq c d_{X}\left(x, x^{\prime}\right)$ for all $x, x^{\prime} \in X$, and bi-Lipschitz if there exists $c>0$ such that $d_{X}\left(x, x^{\prime}\right) / c \leq d_{Y}\left(f(x), f\left(x^{\prime}\right)\right)$ $\leq c d_{X}\left(x, x^{\prime}\right)$ for all $x, x^{\prime} \in X$. The metric space $\left(X, d_{X}\right)$ is Lipschitz equivalent to the metric space $\left(Y, d_{Y}\right)$ if there exists a surjective bi-Lipschitz function $f:\left(X, d_{X}\right) \rightarrow\left(Y, d_{Y}\right)$.

A small scale structure $\mu$ on a set $X$ is a family $\mu$ of covers of $X$ satisfying the following conditions:

- if $\mathcal{U} \in \mu$ and $\mathcal{U}<\mathcal{V}$, then $\mathcal{V} \in \mu$;

- if $\mathcal{U}, \mathcal{V} \in \mu$, then $\mathcal{U} \wedge \mathcal{V} \in \mu$;

- for every $\mathcal{V} \in \mu$, there exists $\mathcal{U} \in \mu$ such that $\mathcal{U}<^{*} \mathcal{V}$;

- for any distinct $x, y \in X$, there exists $\mathcal{U} \in \mu$ such that $(x, y) \nless \mathcal{U}$.

A subfamily $\mu^{\prime}$ of $\mu$ is a base if each $\mathcal{U} \in \mu$ admits $\mathcal{U}^{\prime} \in \mu^{\prime}$ such that $\mathcal{U}^{\prime}<\mathcal{U}$. In this case, $\mu^{\prime}$ is said to generate $\mu$.

Our notion of small scale structure coincides with the notion of uniformity in the sense of J. R. Isbell [6]. The family of all uniform covers of a metric space $(X, d)$ forms a small scale structure. A uniform space $(X, \mu)$ consists of a set $X$ and a small scale structure $\mu$ on $X$.

A large scale structure $\lambda$ on a set $X$ is a non-empty collection $\lambda$ of families of subsets of $X$ satisfying the following conditions:

- if $\mathcal{V} \in \lambda$ and $\mathcal{U}<e(\mathcal{V})$, then $\mathcal{U} \in \lambda$, where the trivial extension e $(\mathcal{U})$ of a family $\mathcal{U}$ is defined as the family $\mathcal{U} \cup\{\{x\}: x \in X\}$;

- if $\mathcal{U}, \mathcal{V} \in \lambda$, then $\operatorname{St}(\mathcal{U}, \mathcal{V}) \in \lambda$;

- for any $x, y \in X$, there exists $\mathcal{U} \in \lambda$ such that $(x, y)<\mathcal{U}$.

A subfamily $\lambda^{\prime}$ of $\lambda$ is a base if each $\mathcal{U} \in \lambda$ admits $\mathcal{U}^{\prime} \in \lambda^{\prime}$ such that $\mathcal{U}<e\left(\mathcal{U}^{\prime}\right)$. In this case, $\lambda^{\prime}$ is said to generate $\lambda$. A coarse space $(X, \lambda)$ consists of a set $X$ with a large scale structure $\lambda$ on $X$.

The definition of large scale structure is due to J. Dydak and C. S. Hoffland [4]; it is equivalent to the notion of coarse structure [10]. The family of all uniformly bounded families of subsets in a metric space $(X, d)$ forms a large scale structure.

The multiplicity of a cover $\mathcal{U}$, denoted $\operatorname{mul}(\mathcal{U})$, is the largest integer $n$ such that there exist $n$ elements of $\mathcal{U}$ with nonempty intersection. 
A uniform space $(X, \mu)$ has large uniform dimension at most $n$, written $\Delta d(X, \mu) \leq n$, if every uniform open cover $\mathcal{U}$ admits a uniform open cover $\mathcal{V}$ such that $\mathcal{V}<\mathcal{U}$ and $\operatorname{mul}(\mathcal{V}) \leq n+1$ (see [6]).

A metrizable space $X$ has covering dimension at most $n$, in symbols $\operatorname{dim} X \leq n$, provided every finite open cover $\mathcal{U}$ admits an open cover $\mathcal{V}$ such that $\mathcal{V}<\mathcal{U}$ and $\operatorname{mul}(\mathcal{V}) \leq n+1$

A coarse space $(X, \lambda)$ has asymptotic dimension at most $n$, written $\operatorname{asdim}(X, \lambda) \leq n$, if every cover $\mathcal{U} \in \lambda$ admits a cover $\mathcal{V} \in \lambda$ such that $\mathcal{U}<\mathcal{V}$ and $\operatorname{mul}(\mathcal{V}) \leq n+1$ (see $[2])$.

Let $\mathbb{R}_{\geq 0}$ denote the set of all non-negative real numbers, and $\mathbb{Z}_{\geq 0}$ the set of all non-negative integers.

3. Metrics induced by normal sequences. For each family $\mathfrak{U}=\left\{\mathcal{U}_{i}\right.$ : $i \in \mathbb{Z}\}$ of covers of a set $X$, consider the following three conditions:

(N1) $\mathcal{U}_{i+1}<^{*} \mathcal{U}_{i}$ for $i \in \mathbb{Z}$.

(N2) For any $x, x^{\prime} \in X$, there exists $i \in \mathbb{Z}$ such that $\left(x, x^{\prime}\right)<\mathcal{U}_{i}$.

(N3) For any distinct $x, x^{\prime} \in X$, there exists $i \in \mathbb{Z}$ such that $\left(x, x^{\prime}\right) \nless \mathcal{U}_{i}$.

For each family $\mathfrak{U}=\left\{\mathcal{U}_{i}: i \in \mathbb{Z}\right\}$ of covers satisfying (N1) and (N2), there is a function $D_{\mathfrak{U}}: X \times X \rightarrow \mathbb{R}_{\geq 0}$ well-defined by

$$
D_{\mathfrak{U}}\left(x, x^{\prime}\right)= \begin{cases}0 & \left(x=x^{\prime}\right), \\ 1 / 3^{\alpha\left(x, x^{\prime}\right)} & \left(x \neq x^{\prime}\right),\end{cases}
$$

where $\alpha\left(x, x^{\prime}\right)=\max \left\{i \in \mathbb{Z}:\left(x, x^{\prime}\right)<\mathcal{U}_{i}\right\}$. Then define $d_{\mathfrak{U}}: X \times X \rightarrow \mathbb{R}_{\geq 0}$ by

$$
d_{\mathfrak{U}}\left(x, x^{\prime}\right)=\inf \left\{D_{\mathfrak{U}}\left(x, x_{1}\right)+D_{\mathfrak{U}}\left(x_{1}, x_{2}\right)+\cdots+D_{\mathfrak{U}}\left(x_{n}, x^{\prime}\right)\right\},
$$

where the infimum runs through all finite sequences $x_{1}, \ldots, x_{n}$ of points in $X$.

Proposition 3.1. The following statements hold:

(1) For each family $\mathfrak{U}=\left\{\mathcal{U}_{i}: i \in \mathbb{Z}\right\}$ of covers satisfying (N1) and (N2), the function $d_{\mathfrak{U}}$ is a pseudometric on $X$, and the following inclusions hold for every $x \in X$ and $i \in \mathbb{Z}$ :

(a) $\operatorname{St}\left(x, \mathcal{U}_{i}\right) \subset \overline{\mathrm{B}}_{d_{\mathfrak{U}}}\left(x, 1 / 3^{i}\right) \subset \mathrm{B}_{d_{\mathfrak{U}}}\left(x, 1 / 3^{i-1}\right)$, and

(b) $\overline{\mathrm{B}}_{d_{\mathfrak{U}}}\left(x, 1 / 3^{i}\right) \subset \operatorname{St}\left(x, \mathcal{U}_{i-2}\right)$.

(2) If $\mathfrak{U}$ additionally satisfies (N3), then $d_{\mathfrak{U}}$ is a metric on $X$.

Proof. That $d_{\mathfrak{U}}$ is a pseudometric easily follows from the definition. The inclusions (a) and (b) in (1) follow from the inequalities $d_{\mathfrak{U}}\left(x, x^{\prime}\right) \leq D_{\mathfrak{U}}\left(x, x^{\prime}\right)$ $\leq 6 d_{\mathfrak{U}}\left(x, x^{\prime}\right)$ for $x, x^{\prime} \in X$ (proven analogously to [8, 2-16]). To see (2), for any distinct $x, x^{\prime} \in X$, there exists $i \in \mathbb{Z}$ such that $\left(x, x^{\prime}\right) \nless \mathcal{U}_{i}$. This together with (b) implies $d_{\mathfrak{U}}\left(x, x^{\prime}\right)>1 / 3^{i+2}$, showing that $d_{\mathfrak{U}}$ defines a metric on $X$. 
A family $\mathfrak{U}=\left\{\mathcal{U}_{i}: i \in \mathbb{Z}\right\}$ of covers of $X$ is called a normal sequence if it satisfies conditions (N1)-(N3).

Proposition 3.2. Let $(X, d)$ be a metric space, and let $\mathfrak{U}=\left\{\mathcal{U}_{i}: i \in \mathbb{Z}\right\}$ be the normal sequence consisting of the covers $\mathcal{U}_{i}$ of $X$ by the open $1 / 3^{i}$ balls. Then:

(1) $d_{\mathfrak{U}}\left(x, x^{\prime}\right) / 3 \leq d\left(x, x^{\prime}\right)<81 d_{\mathfrak{U}}\left(x, x^{\prime}\right)$ for $x, x^{\prime} \in X$.

(2) The metric space $\left(X, d_{\mathfrak{U}}\right)$ is Lipschitz equivalent to $(X, d)$.

Proof. It suffices to verify (1). Let $x, x^{\prime} \in X$, and let $i, j \in \mathbb{Z}$ be such that $1 / 3^{i+1}<d\left(x, x^{\prime}\right) \leq 1 / 3^{i}$ and $1 / 3^{j+1}<d_{\mathfrak{U}}\left(x, x^{\prime}\right) \leq 1 / 3^{j}$. Then $d_{\mathfrak{U}}\left(x, x^{\prime}\right) \leq 1 / 3^{i} \leq 3 d\left(x, x^{\prime}\right)$ and $d\left(x, x^{\prime}\right) \leq 2 / 3^{j-2}<81 d_{\mathfrak{U}}\left(x, x^{\prime}\right)$ (see Proposition $3.1(1))$.

Our notion of normal sequence demonstrates the micro-macro analogy between small and large scales. The notion of normal sequence unifies the notions of Čech approximation in small scale and anti-Čech approximation in large scale. A sequence $\mathfrak{U}=\left\{\mathcal{U}_{i}: i \in \mathbb{Z}\right\}$ of covers satisfying the condition St $\mathcal{U}_{i+1}<\mathcal{U}_{i}$ is a normal sequence if the positive part $\left\{\mathcal{U}_{i}: i \geq 0\right\}$ and the negative part $\left\{\mathcal{U}_{-i}: i \leq 0\right\}$ are a Čech approximation and an anti-Čech approximation, respectively.

4. Lipschitz functions. In this section, we consider three types of Lipschitz function: large scale Lipschitz functions, small scale Lipschitz functions, and locally Lipschitz functions. We characterize each type of Lipschitz function in terms of a normal sequence.

Definition 4.1. A function $f:\left(X, d_{X}\right) \rightarrow\left(Y, d_{Y}\right)$ between metric spaces is large scale Lipschitz if there exist $c, d>0$ such that

$$
d_{Y}\left(f(x), f\left(x^{\prime}\right)\right) \leq c d_{X}\left(x, x^{\prime}\right)+d \quad \text { for all } x, x^{\prime} \in X .
$$

A function $f:\left(X, d_{X}\right) \rightarrow\left(Y, d_{Y}\right)$ is a quasi-isometry if there exist $c \geq 1$ and $d>0$ such that

$$
d_{X}\left(x, x^{\prime}\right) / c-d \leq d_{Y}\left(f(x), f\left(x^{\prime}\right)\right) \leq c d_{X}\left(x, x^{\prime}\right)+d \quad \text { for all } x, x^{\prime} \in X
$$

and the image $f(X)$ is a net in $Y$, i.e., there exists $M>0$ such that for each $y \in Y$, there exists $x \in X$ with $d_{Y}(f(x), y)<M$. Two metric spaces $\left(X, d_{X}\right)$ and $\left(Y, d_{Y}\right)$ are quasi-isometric if there exists a quasi-isometry $f$ : $\left(X, d_{X}\right) \rightarrow\left(Y, d_{Y}\right)$.

The following is the definition of small scale Lipschitz function, a notion dual to large scale Lipschitz function.

Definition 4.2. A function $f:\left(X, d_{X}\right) \rightarrow\left(Y, d_{Y}\right)$ is small scale Lipschitz if there exist $c, r>0$ such that

$$
d_{Y}\left(f(x), f\left(x^{\prime}\right)\right) \leq c d_{X}(x, y) \quad \text { for all } x, x^{\prime} \in X \text { with } d_{X}\left(x, x^{\prime}\right)<r,
$$


and $f$ is a small scale Lipschitz equivalence if $f$ is bijective and both $f$ and $f^{-1}$ are small scale Lipschitz. Two metric spaces $\left(X, d_{X}\right)$ and $\left(Y, d_{Y}\right)$ are small scale Lipschitz equivalent if there exists a small scale Lipschitz equivalence $f:\left(X, d_{X}\right) \rightarrow\left(Y, d_{Y}\right)$.

We recall the definition of local Lipschitz function.

Definition 4.3. A function $f:\left(X, d_{X}\right) \rightarrow\left(Y, d_{Y}\right)$ is locally Lipschitz if for each $x \in X$, there exist $c, \varepsilon>0$ such that $f \mid B(x, \varepsilon)$ is a Lipschitz function, and $f$ is a lipeomorphism if $f$ is bijective and both $f$ and $f^{-1}$ are locally Lipschitz. Two metric spaces $\left(X, d_{X}\right)$ and $\left(Y, d_{Y}\right)$ are lipeomorphic if there exists a lipeomorphism $f:\left(X, d_{X}\right) \rightarrow\left(Y, d_{Y}\right)$.

EXAMPLE 4.4. It can be proven that every locally Lipschitz function on a compact space is Lipschitz. Clearly, every small scale Lipschitz function is locally Lipschitz. The function $f:[0, \infty) \rightarrow[0, \infty)$ defined by $f(x)=\sqrt{x}$ is large scale Lipschitz but not locally Lipschitz at 0 . The function $g: \mathbb{R} \rightarrow \mathbb{R}$ defined by $g(x)=x^{2}$ is locally Lipschitz but neither small scale Lipschitz nor large scale Lipschitz. The function $h: \mathbb{N} \rightarrow \mathbb{R}$ defined by $h(x)=x^{2}$ is small scale Lipschitz but not large scale Lipschitz.

The three types of Lipschitz functions are characterized by normal sequences.

Proposition 4.5. Let $d$ and $d^{\prime}$ be two metrics on a set $X$, and let $\mathcal{U}_{i}$ and $\mathcal{U}_{i}^{\prime}$ be the covers by the open $1 / 3^{i}$-balls with respect to the metrics $d$ and $d^{\prime}$, respectively. Then:

(1) The metric spaces $(X, d)$ and $\left(X, d^{\prime}\right)$ are quasi-isometric if and only if there exist $m \in \mathbb{Z}_{\geq 0}$ and $i_{0} \in \mathbb{Z}$ such that $\mathcal{U}_{i}<\mathcal{U}_{i-m}^{\prime}$ and $\mathcal{U}_{i}^{\prime}<\mathcal{U}_{i-m}$ for each $i \leq i_{0}$.

(2) $(X, d)$ and $\left(X, d^{\prime}\right)$ are small scale Lipschitz equivalent if and only if there exist $m \in \mathbb{Z}_{\geq 0}$ and $i_{0} \in \mathbb{Z}$ such that $\mathcal{U}_{i+m}<\mathcal{U}_{i}^{\prime}$ and $\mathcal{U}_{i+m}^{\prime}<\mathcal{U}_{i}$ for each $i \geq i_{0}$.

(3) $(X, d)$ and $\left(X, d^{\prime}\right)$ are lipeomorphic if and only if for each $x \in X$, there exist $m \in \mathbb{Z}_{\geq 0}$ and $i_{0} \in \mathbb{Z}$ such that $\operatorname{St}\left(x, \mathcal{U}_{i+m}\right) \subset \operatorname{St}\left(x, \mathcal{U}_{i}\right)$ and $\operatorname{St}\left(x, \mathcal{U}_{i+m}^{\prime}\right) \subset \operatorname{St}\left(x, \mathcal{U}_{i}\right)$ for each $i \geq i_{0}$.

Proof. (1) Suppose that there exist $c, d>0$ such that $d^{\prime}\left(x, x^{\prime}\right) / c-d \leq$ $d\left(x, x^{\prime}\right) \leq c d\left(x, x^{\prime}\right)+d$ for any $x, x^{\prime} \in X$. Take $m \in \mathbb{Z}_{\geq 0}$ and $i_{0} \in \mathbb{Z}$ such that $c \leq 3^{m-1}$ and $d \leq 1 / 3^{i_{0}+1}$, respectively. Then $\mathcal{U}_{i+m}<\mathcal{U}_{i}^{\prime}$ and $\mathcal{U}_{i+m}^{\prime}<\mathcal{U}_{i}$ for $i \leq i_{0}$. Indeed, to see the first refinement, note that $d\left(x, x^{\prime}\right)<1 / 3^{i+m}$ implies

$$
d^{\prime}\left(x, x^{\prime}\right) \leq c d\left(x, x^{\prime}\right)+d<3^{m-1} \cdot 1 / 3^{i+m}+1 / 3^{i_{0}+1} \leq 2 / 3^{i+1}<1 / 3^{i} .
$$

The other refinement can be verified similarly. 
Conversely, suppose that there exist $m \in \mathbb{Z}_{\geq 0}$ and $i_{0} \in \mathbb{Z}$ such that $\mathcal{U}_{i+m}<\mathcal{U}_{i}^{\prime}$ and $\mathcal{U}_{i+m}^{\prime}<\mathcal{U}_{i}$ for each $i \leq i_{0}$. Let $c=2 \cdot 3^{m+1}$ and $d=2 / 3^{i_{0}}$, and let $x, x^{\prime} \in X$. If $d\left(x, x^{\prime}\right) \geq 1 / 3^{i_{0}+m+1}$, let $i \leq i_{0}$ be the unique integer such that $1 / 3^{i+m+1} \leq d\left(x, x^{\prime}\right)<1 / 3^{i+m}$; then $d^{\prime}\left(x, x^{\prime}\right)<2 \cdot 1 / 3^{i}=$ $2 \cdot 3^{m+1} / 3^{i+m+1} \leq c d\left(x, x^{\prime}\right)$. If $d\left(x, x^{\prime}\right)<1 / 3^{i_{0}+m+1}$, then this implies $d^{\prime}\left(x, x^{\prime}\right)<d$. Thus $d^{\prime}\left(x, x^{\prime}\right) \leq c d\left(x, x^{\prime}\right)+d$. The other inequality can be verified similarly.

(2) Suppose that there exist $c, r>0$ such that $d^{\prime}\left(x, x^{\prime}\right) \leq c d\left(x, x^{\prime}\right)$ for any $x, x^{\prime} \in X$ with $d\left(x, x^{\prime}\right)<r$, and $d\left(x, x^{\prime}\right) \leq c d^{\prime}\left(x, x^{\prime}\right)$ for any $x, x^{\prime} \in X$ with $d^{\prime}\left(x, x^{\prime}\right)<r$. Take $m \in \mathbb{Z}_{\geq 0}$ such that $c \leq 3^{m}$, and then $i_{0} \in \mathbb{Z}$ such that $1 / 3^{i_{0}+m} \leq r$. Then $\mathcal{U}_{i+m}<\mathcal{U}_{i}^{\prime}$ for $i \geq i_{0}$ since $d\left(x, x^{\prime}\right)<1 / 3^{i+m}$ implies $d^{\prime}\left(x, x^{\prime}\right)<c / 3^{i+m} \leq 1 / 3^{i}$. Similarly, $\mathcal{U}_{i+m}^{\prime}<\mathcal{U}_{i}$.

Conversely, suppose that there exist $m \in \mathbb{Z}_{\geq 0}$ and $i_{0} \in \mathbb{Z}$ such that $\mathcal{U}_{i+m}<\mathcal{U}_{i}^{\prime}$ and $\mathcal{U}_{i+m}^{\prime}<\mathcal{U}_{i}$ for each $i \geq i_{0}$. Take $r$ such that $0<r$ $<1 / 3^{i_{0}+m}$, and let $x, x^{\prime} \in X$ satisfy $d\left(x, x^{\prime}\right)<r$. Then, for each $s>0$ with $d_{\mathfrak{U}}\left(x, x^{\prime}\right)<s$, there exist finitely many points $x_{0}=x, x_{1}, \ldots, x_{n+1}=x^{\prime}$ such that $\sum_{j=0}^{n} D_{\mathfrak{U}}\left(x_{j}, x_{j+1}\right)<s$ and $D_{\mathfrak{U}}\left(x_{j}, x_{j+1}\right)=1 / 3^{k_{j}+m}$ for some $k_{j} \geq i_{0}$. Since $\mathcal{U}_{k_{j}+m}<\mathcal{U}_{k_{j}}^{\prime}$, we get $D_{\mathfrak{U}^{\prime}}\left(x_{j}, x_{j+1}\right) \leq 1 / 3^{k_{j}}=3^{m} D_{\mathfrak{U}}\left(x_{j}, x_{j+1}\right)$. This implies $d_{\mathfrak{U}^{\prime}}\left(x, x^{\prime}\right) \leq 3^{m} \sum_{j=0}^{n} D_{\mathfrak{U}}\left(x_{j}, x_{j+1}\right)<3^{m} s$, and hence $d_{\mathfrak{U}^{\prime}}\left(x, x^{\prime}\right) \leq$ $3^{m} d_{\mathfrak{U}}\left(x, x^{\prime}\right)$. This together with Proposition $3.2(1)$ implies $d^{\prime}\left(x, x^{\prime}\right) \leq$ $3^{m+4} d\left(x, x^{\prime}\right)$ for any $x, x^{\prime} \in X$ with $d\left(x, x^{\prime}\right)<r$. The opposite inequality $d\left(x, x^{\prime}\right) \leq 3^{m+4} d^{\prime}\left(x, x^{\prime}\right)$ is proven similarly.

(3) is proven by the same argument as for (1).

Normal sequences $\mathcal{U}=\left\{\mathcal{U}_{i}: i \in \mathbb{Z}\right\}$ and $\mathcal{U}^{\prime}=\left\{\mathcal{U}_{i}^{\prime}: i \in \mathbb{Z}\right\}$ on a set $X$ are said to be quasi-isometric, small scale Lipschitz equivalent or lipeomorphic if they satisfy the conditions in (1), (2) and (3) of Proposition 4.5, respectively. Proposition 3.2 immediately implies

Corollary 4.6. Let $\mathcal{U}=\left\{\mathcal{U}_{i}: i \in \mathbb{Z}\right\}$ be a normal sequence on a set $X$, and let $\mathcal{U}^{\prime}=\left\{\mathcal{U}_{i}^{\prime}: i \in \mathbb{Z}\right\}$ be the normal sequence consisting of the covers $\mathcal{U}_{i}^{\prime}$ of $X$ by the open $1 / 3^{i}$-balls with respect to the metric $d_{\mathfrak{U}}$. Then $\mathfrak{U}$ and $\mathfrak{U}^{\prime}$ are quasi-isometric, small scale Lipschitz equivalent and lipeomorphic.

5. Metrization and normal sequences. N. Wright [11] defined a set $X$ with both a topological structure and a large scale structure to be $s i$ multaneously metrizable if there exists a metric $d$ on $X$ which induces both structures. Analogously, we define a set $X$ with both a small scale structure and a large scale structure to be simultaneously metrizable if there exists a metric $d$ on $X$ which induces both structures.

The following theorem gives a necessary and sufficient condition on normal sequences for a set with a small scale structure and a large scale structure to be simultaneously metrizable. 
THEOREM 5.1. Let $X$ be a set with both a small scale structure and a large scale structure. Then $X$ is simultaneously metrizable if and only if

(1) $\left\{\mathcal{U}_{i}: i \leq 0\right\}$ generates the large scale structure, and

(2) $\left\{\mathcal{U}_{i}: i \geq 0\right\}$ generates the small scale structure.

Proof. Suppose that $(X, d)$ is a metric space which induces the given small scale and large scale structures. For each $i \in \mathbb{Z}$, let $\mathcal{U}_{i}$ be the cover consisting of the open $1 / 3^{i}$-balls. Then $\left\{\mathcal{U}_{i}: i \in \mathbb{Z}\right\}$ is a normal sequence satisfying (1) and (2).

Conversely, suppose that a normal sequence $\mathfrak{U}=\left\{\mathcal{U}_{i}: i \in \mathbb{Z}\right\}$ satisfies (1) and (2). Then $\mathfrak{U}$ induces a metric $d_{\mathfrak{U}}$ satisfying (a) and (b) in Proposition $3.1(1)$. If $\mathcal{B}_{i}$ is the cover by the open $1 / 3^{i}$-balls in $\left(X, d_{\mathfrak{U}}\right)$, then $\mathcal{U}_{i}<\mathcal{B}_{i-1}$ and $\mathcal{B}_{i}<\mathcal{U}_{i-3}$. This shows that $d_{\mathfrak{U}}$ induces the small scale and large scale structures generated by $\mathfrak{U}$.

The following theorem, which is proven by a similar argument, gives a necessary and sufficient condition on normal sequences for a set with both a topological structure and a large scale structure to be simultaneously metrizable.

THEOREM 5.2. Let $X$ be a set with both a topological structure and a large scale structure. Then $X$ is simultaneously metrizable if and only if there exists a normal sequence $\mathfrak{U}=\left\{\mathcal{U}_{i}: i \in \mathbb{Z}\right\}$ on $X$ such that

(1) $\left\{\mathcal{U}_{i}: i \leq 0\right\}$ generates the large scale structure, and

(2) for each $x \in X$, the family $\left\{\operatorname{St}\left(x, \mathcal{U}_{i}\right): i \geq 0\right\}$ is a base at $x$.

The following two theorems are variations of Theorems 5.1 and 5.2 .

THEOREM 5.3. Let $X$ be a set with both a small scale structure $\mu$ and a large scale structure $\lambda$. Then $X$ is simultaneously metrizable and satisfies $\Delta d(X, \mu) \leq n$ and $\operatorname{asdim}(X, \lambda) \leq m$ if and only if $X$ admits a normal sequence $\mathfrak{U}=\left\{\mathcal{U}_{i}: i \in \mathbb{Z}\right\}$ such that

(1) $\left\{\mathcal{U}_{i}: i \leq 0\right\}$ generates $\lambda$ and $\operatorname{mul}\left(\mathcal{U}_{i}\right) \leq m+1$ for $i \leq 0$, and

(2) $\left\{\mathcal{U}_{i}: i \geq 0\right\}$ generates $\mu$, and $\operatorname{mul}\left(\mathcal{U}_{i}\right) \leq n+1$ for $i \geq 0$.

Proof. Suppose that $d$ is a metric which induces the given small scale and large scale structures. Let $\mathcal{U}_{0}$ be the cover by open 1-balls. Then $\mathcal{U}_{0} \in \mu$ and $\mathcal{U}_{0} \in \lambda$. As $\Delta d(X, \mu) \leq n$, we can inductively find covers $\mathcal{U}_{i} \in \mu$ for $i \geq 0$ such that $\mathcal{U}_{i+1}<^{*} \mathcal{U}_{i}, \operatorname{mul}\left(\mathcal{U}_{i}\right) \leq n+1$ and $\operatorname{mesh}_{d}\left(\mathcal{U}_{i}\right) \leq 1 / 3^{i}$. Since $\operatorname{asdim}(X, \lambda) \leq m$, we can inductively find covers $\mathcal{U}_{i} \in \mu$ for $i<0$ such that $\mathcal{U}_{i+1}<^{*} \mathcal{U}_{i}, \operatorname{mul}\left(\mathcal{U}_{i}\right) \leq m+1$ and $\operatorname{Leb}_{d}\left(\mathcal{U}_{i}\right) \geq 1 / 3^{i}$. Then $\mathfrak{U}=\left\{\mathcal{U}_{i}: i \in \mathbb{Z}\right\}$ is a normal sequence on $X$ which satisfies (1) and (2).

Conversely, suppose that a normal sequence $\mathfrak{U}=\left\{\mathcal{U}_{i}: i \in \mathbb{Z}\right\}$ satisfies (1) and (2). By the same argument as in the proof of Theorem 5.1, the metric $d_{\mathfrak{U}}$ induced by $\mathfrak{U}$ induces the small scale and large scale structures generated 
by $\mathfrak{U}$. Conditions (1) and (2) imply $\Delta d(X, \mu) \leq n$ and $\operatorname{asdim}(X, \lambda) \leq m$, respectively.

THEOREM 5.4. Let $X$ be a set with both a topological structure $\tau$ and a large scale structure $\lambda$. Then $X$ is simultaneously metrizable and satisfies $\operatorname{dim}(X, \tau) \leq n$ and $\operatorname{asdim}(X, \lambda) \leq m$ if and only if $X$ admits a normal sequence $\mathfrak{U}=\left\{\mathcal{U}_{i}: i \in \mathbb{Z}\right\}$ such that

(1) $\left\{\mathcal{U}_{i}: i \leq 0\right\}$ generates $\lambda$ and $\operatorname{mul}\left(\mathcal{U}_{i}\right) \leq m+1$ for $i \leq 0$, and

(2) for each $x \in X$, the family $\left\{\operatorname{St}\left(x, \mathcal{U}_{i}\right): i \geq 0\right\}$ is a base at $x$, and $\operatorname{mul}\left(\mathcal{U}_{i}\right) \leq n+1$ for $i \geq 0$.

Proof. The proof is similar to that of Theorem 5.3 .

6. Compatibility of small and large scale structures. Given a set $X$ equipped with a topological structure and a coarse structure, Wright [11] defined the notion of compatibility of the two structures: the topological structure is compatible with the coarse structure if every entourage is contained in an open entourage, equivalently, there exists an open entourage containing the diagonal. Wright proved

TheOREM 6.1. Let $X$ be a set with metrics $d_{T}$ and $d_{L}$. If the topological structure $\tau$ induced by $d_{T}$ is compatible with the large scale structure $\lambda$ induced by $d_{L}$, then there exists a metric $d$ such that the metric space $(X, d)$ is homeomorphic to $\left(X, d_{T}\right)$ and quasi-isometric to $\left(X, d_{L}\right)$.

In this section, extending this definition, we define compatibility of a small scale structure and a large scale structure. For any set $X$ equipped with a small scale structure $\mu$ and a large scale structure $\lambda, \mu$ is said to be compatible with $\lambda$ if there exists a cover $\mathcal{U}$ such that $\mathcal{U} \in \mu$ and $\mathcal{U} \in \lambda$. For any set $X$ equipped with a topological structure $\tau$ and a large scale structure $\lambda, \tau$ is said to be compatible with $\lambda$ if there exists a cover $\mathcal{U}$ such that each element of $\mathcal{U}$ is open in $\tau$ and $\mathcal{U} \in \lambda$. It is easy to see that $\tau$ is compatible with $\lambda$ in our sense if and only if $\tau$ is compatible with the coarse structure induced by $\lambda$ in the sense of [11].

In this section, we show the following two theorems:

TheOREM 6.2. Let $X$ be a set with metrics $d_{S}$ and $d_{L}$. If the small scale structure $\mu$ induced by $d_{S}$ is compatible with the large scale structure $\lambda$ induced by $d_{L}$, then there exists a metric $d$ such that the metric space $(X, d)$ is small scale Lipschitz equivalent to $\left(X, d_{S}\right)$ and quasi-isometric to $\left(X, d_{L}\right)$.

TheOREm 6.3. Let $X$ be a set with metrics $d_{T}$ and $d_{L}$. If the topological structure $\tau$ induced by $d_{T}$ is compatible with the large scale structure $\lambda$ induced by $d_{L}$, then there exists a metric d such that $(X, d)$ is lipeomorphic to $\left(X, d_{T}\right)$ and quasi-isometric to $\left(X, d_{T}\right)$. 
Proof of Theorem 6.2. Let $\mathcal{U}$ be a cover of $X$ such that $\mathcal{U} \in \mu$ and $\mathcal{U} \in \lambda$, and take $i_{0}>0$ such that $2 / 3^{i_{0}}<\operatorname{Leb}_{d_{T}}(\mathcal{U})$, and $j_{0}<0$ such that $\operatorname{mesh}_{d_{L}}(\mathcal{U})<1 / 3^{j_{0}}$. For each $i \geq 0$, let $\mathcal{U}_{i}$ be the cover consisting of the open $1 / 3^{i+i_{0}}$-balls in $\left(X, d_{T}\right)$, and for each $i<0$, let $\mathcal{U}_{i}$ be the cover consisting of the open $1 / 3^{i+j_{0}}$-balls in $\left(X, d_{L}\right)$. Then $\mathcal{U}_{0}<\mathcal{U}$ and $\mathcal{U}<^{*} \mathcal{U}_{-1}$, so $\mathcal{U}_{0}<^{*} \mathcal{U}_{-1}$. Thus $\mathfrak{U}=\left\{\mathcal{U}_{i}: i \in \mathbb{Z}\right\}$ is a normal sequence, and induces the metric $d_{\mathfrak{U}}$ on $X$. If, for each $i \in \mathbb{Z}, \mathcal{U}_{i}^{\prime}$ is the cover by the open $1 / 3^{i}$-balls with respect to $d_{\mathfrak{U}}$, then (a) and (b) in Proposition 3.1(1) imply $\mathcal{U}_{i+1}<\mathcal{U}_{i}^{\prime}$ and $\mathcal{U}_{i+3}^{\prime}<\mathcal{U}_{i}$. This together with Proposition 4.5(1)\&(2) implies that $\left(X, d_{\mathfrak{U}}\right)$ is quasi-isometric to $\left(X, d_{L}\right)$ and small scale Lipschitz equivalent to $\left(X, d_{S}\right)$ as required.

Proof of Theorem 6.3. Let $\mathcal{U}$ be an open cover of $X$ such that $\mathcal{U} \in \lambda$. Then there exists a sequence of open covers $\mathcal{U}_{i}$ for $i \geq 0$ consisting of open balls in $\left(X, d_{T}\right)$ such that $\operatorname{mesh}_{d_{T}}\left(\mathcal{U}_{i}\right)<1 / 3^{i}, \mathcal{U}_{0}<^{*} \mathcal{U}$ and $\mathcal{U}_{i+1}<^{*} \mathcal{U}_{i}$. There also exists a sequence of open covers $\mathcal{U}_{i}$ for $i<0$ consisting of open balls in $\left(X, d_{L}\right)$ such that $\operatorname{Leb}_{d_{L}}\left(\mathcal{U}_{i}\right)>1 / 3^{i}, \mathcal{U}<\mathcal{U}_{-1}$ and $\mathcal{U}_{i}<\mathcal{U}_{i-1}$. Then $\mathfrak{U}=\left\{\mathcal{U}_{i}: i \in \mathbb{Z}\right\}$ is a normal sequence and induces the metric $d_{\mathfrak{U}}$ on $X$. Let $\mathcal{U}_{i}^{\prime}$ be the cover by the open $1 / 3^{i}$-balls in $\left(X, d_{\mathfrak{U}}\right)$ for $i \in \mathbb{Z}$. Then (a) and (b) in Proposition 3.1(1) imply $\operatorname{St}\left(x, \mathcal{U}_{i+1}\right) \subset \operatorname{St}\left(x, \mathcal{U}_{i}^{\prime}\right)$ and $\operatorname{St}\left(x, \mathcal{U}_{i+3}^{\prime}\right) \subset$ $\operatorname{St}\left(x, \mathcal{U}_{i}\right)$ for each $i \in \mathbb{Z}$. This together with Proposition 4.5(1)\&(3) implies that $\left(X, d_{\mathfrak{U}}\right)$ is quasi-isometric to $\left(X, d_{L}\right)$ and lipeomorphic to $\left(X, d_{T}\right)$.

The following two theorems concern the problem of simultaneous ultrametrization for a set with a 0-dimensional small scale structure and a 0-dimensional large scale structure.

TheOREM 6.4. Let $X$ be a set with metrics $d_{S}$ and $d_{L}$, and equip it with the small scale structure $\mu$ induced by $d_{S}$ and the large scale structure $\lambda$ induced by $d_{L}$. Suppose that $\tau$ is compatible with $\lambda$. Then $\Delta d(X, \mu)=0$ and $\operatorname{asdim}(X, \lambda)=0$ if and only if there exists an ultrametric $d$ on $X$ such that the metric space $(X, d)$ is small scale Lipschitz equivalent to $\left(X, d_{S}\right)$ and quasi-isometric to $\left(X, d_{L}\right)$.

Proof. There exists a cover $\mathcal{U}$ of $X$ such that $\mathcal{U} \in \mu$ and $\mathcal{U} \in \lambda$. Let $\mathcal{U}_{0}=\mathcal{U}$. Then $\Delta d(X, \mu)=0$ implies that there exist $\mathcal{U}_{i} \in \mu$ for $i \geq 0$ such that $\mathcal{U}_{i+1}<^{*} \mathcal{U}_{i}, \operatorname{mul}\left(\mathcal{U}_{i}\right) \leq 1$ and $\operatorname{mesh}_{d_{S}}\left(\mathcal{U}_{i}\right) \leq 1 / 3^{i}$. Then $\operatorname{asdim}(X, \lambda)=0$ implies that there exist $\mathcal{U}_{i} \in \mu$ for $i<0$ such that $\mathcal{U}_{i+1}<^{*} \mathcal{U}_{i}, \operatorname{mul}\left(\mathcal{U}_{i}\right) \leq 1$ and $\operatorname{Leb}_{d_{L}}\left(\mathcal{U}_{i}\right) \geq 1 / 3^{i}$. Then $\mathfrak{U}=\left\{\mathcal{U}_{i}: i \in \mathbb{Z}\right\}$ is a normal sequence on $X$, and it induces the metric $d_{\mathfrak{U}}$. The condition $\operatorname{mul}\left(\mathcal{U}_{i}\right) \leq 1$ implies that $d_{\mathfrak{U}}=D_{\mathfrak{U}}$. To see that this is an ultrametric, let $x, y, z \in X$, and suppose $d_{\mathfrak{U}}(x, y)=1 / 3^{i}$ and $d_{\mathfrak{U}}(y, z)=1 / 3^{j}$. Without loss of generality, assume $i \leq j$. Then there exist $U, V \in \mathcal{U}_{i}$ such that $x, y \in U$ and $y, z \in V$. Since $\operatorname{mul}\left(\mathcal{U}_{i}\right) \leq 1$ and $U \cap V \neq \emptyset$, we have $x, z \in U=V$, which implies

$$
d_{\mathfrak{U}}(x, y)=D_{\mathfrak{U}}(x, z) \leq 1 / 3^{i}=\max \left\{d_{\mathfrak{U}}(x, y), d_{\mathfrak{U}}(y, z)\right\} .
$$


Similarly to the proof of Theorem 6.2, Proposition 4.5(1)\&(2) implies that $(X, d)$ is quasi-isometric to $\left(X, d_{L}\right)$ and small scale Lipschitz equivalent to $\left(X, d_{S}\right)$.

Similarly to Theorem 6.4, we can prove

TheOREM 6.5. Let $X$ be a set with metrics $d_{T}$ and $d_{L}$, and equip it with the topological structure $\tau$ induced by $d_{T}$ and the large scale structure $\lambda$ induced by $d_{L}$. Then $\operatorname{dim}(X, \tau)=0$ and $\operatorname{asdim}(X, \lambda)=0$ if and only if there exists a ultrametric $d$ on $X$ such that the metric $(X, d)$ is lipeomorphic to $\left(X, d_{T}\right)$ and quasi-isometric to $\left(X, d_{L}\right)$.

\section{References}

[1] P. Alexandroff et P. Urysohn, Une condition nécessaire et suffisante pour qu'une classe (L) soit une classe (B), C. R. Acad. Sci. Paris Sér. A-B 177 (1923), 1274-1276.

[2] G. Bell and A. Dranishnikov, Asymptotic dimension in Będlewo, Topology Proc. 38 (2011), 209-236.

[3] N. Brodskiy, J. Dydak, J. Higes, and A. Mitra, Dimension zero at all scales, Topology Appl. 154 (2007), 2729-2740.

[4] J. Dydak and C. S. Hoffland, An alternative definition of coarse structures, Topology Appl. 155 (2008), 1013-1021.

[5] J. de Groot, On a metric that characterizes dimension, Canad. J. Math. 9 (1957), $511-514$.

[6] J. R. Isbell, Uniform Spaces, Amer. Math. Soc., Providence, RI, 1964.

[7] T. Miyata and Ž. Virk, Dimension-raising maps in a large scale, Fund. Math. 223 (2013), 83-97.

[8] K. Nagami, Dimension Theory, Academic Press, 1970.

[9] J. Nagata, On a relation between dimension and metrization, Proc. Japan Acad. 32 (1956), 237-240.

[10] J. Roe, Lectures on Coarse Geometry, Univ. Lecture Ser. 31, Amer. Math. Soc., Providence, RI, 2003.

[11] N. Wright, Simultaneous metrizability of coarse spaces, Proc. Amer. Math. Soc. 139 (2011), 3271-3278.

Takahisa Miyata

Department of Mathematics and Informatics

Graduate School of Human Development and Environment

Kobe University

Kobe, 657-8501 Japan

E-mail: tmiyata@kobe-u.ac.jp 